EPJ Web of Conferences 41, 06005 (2013)

DOI: $10.1051 /$ epjconf/20134106005

C) Owned by the authors, published by EDP Sciences, 2013

\title{
Azide-water intermolecular coupling measured by 2-color 2D IR spectroscopy
}

\author{
J. A. Borek ${ }^{1}$, F. Perakis ${ }^{1}$ and P. Hamm ${ }^{1}$ \\ ${ }^{1}$ Physical Chemistry Institute, University of Zürich, Winterthurerstr. 190, 8057 Zürich, Switzerland
}

\begin{abstract}
We present 2-color two-dimensional infrared spectroscopy of intermolecular coupling between azide ions and their solvation shell water molecules. The cross-peak between azide asymmetric stretch vibration and the OD-stretch vibration is a result of low-probability uphill population transfer. Narrow bleach/excited state absorption peak shows selectivity to solvation shell water molecules only and the characteristics of the cross-peak suggest that the solvation shell hydrogen bond potential has similar anharmonic properties as the hydrogen bond in ice Ih. Population and depopulation of the excited state of the OD-stretch vibration happen on $150 \mathrm{fs}$ and $1.7 \mathrm{ps}$ timescales, respectively, with early manifesting heating effects that limit the selectivity to population times up to $1 \mathrm{ps}$.
\end{abstract}

\section{Introduction}

Two-dimensional infrared spectroscopy is a useful tool to analyze ultrafast vibrational dynamics and vibrational couplings in a variety of systems, including the hydrogen bond network in liquid water and in solutions [1,2]. This is since the hydroxyl stretch has been directly associated with hydrogen bonding strength and thus providing a means to observe the bond strength between the ion and water. Therefore it is a suitable 'sensor' for any rearrangement within the ion's solvation shell.

By utilising two-color 2D IR one can make use of coupling between a solute and its aqueous solvation shell to selectively probe the solvation shell water molecules only, independently of spectral overlap between bulk and solvation shell water [3]. In our experiments, we pump the asymmetric stretch vibration of azide ion $\left(\mathrm{N}_{3}{ }^{-}\right)$and probe the OD-stretch vibration of $\mathrm{D}_{2} \mathrm{O}$ in a $1 \mathrm{M}$ $\mathrm{N}_{3}^{-}$in $\mathrm{D}_{2} \mathrm{O}$ solution and hence observe a cross-peak between those two vibrations in the 2D IR spectra [4]. This allows us to directly probe only the OD-stretch vibration of those $\mathrm{D}_{2} \mathrm{O}$ molecules directly coupled to azide, hence the ion's solvation shell only.

The above-mentioned approach is advantageous, since it does not require a spectral distinction between bulk and solvation shell water and can be applied to a variety of samples, not only the ones in which the hydroxyl stretch vibrational band splits upon solvation.

This is an Open Access article distributed under the terms of the Creative Commons Attribution License 2.0, which permits unrestricted use, distribution, and reproduction in any medium, provided the original work is properly cited. 
A
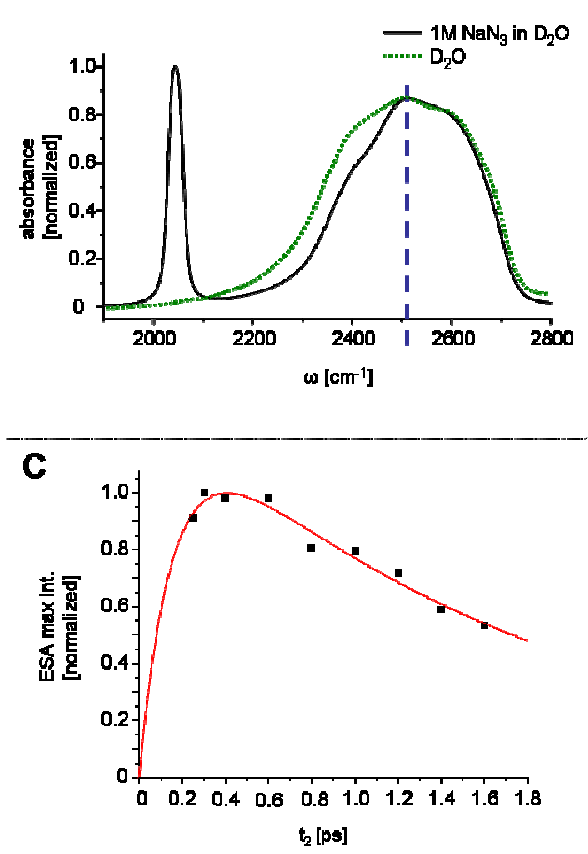

B
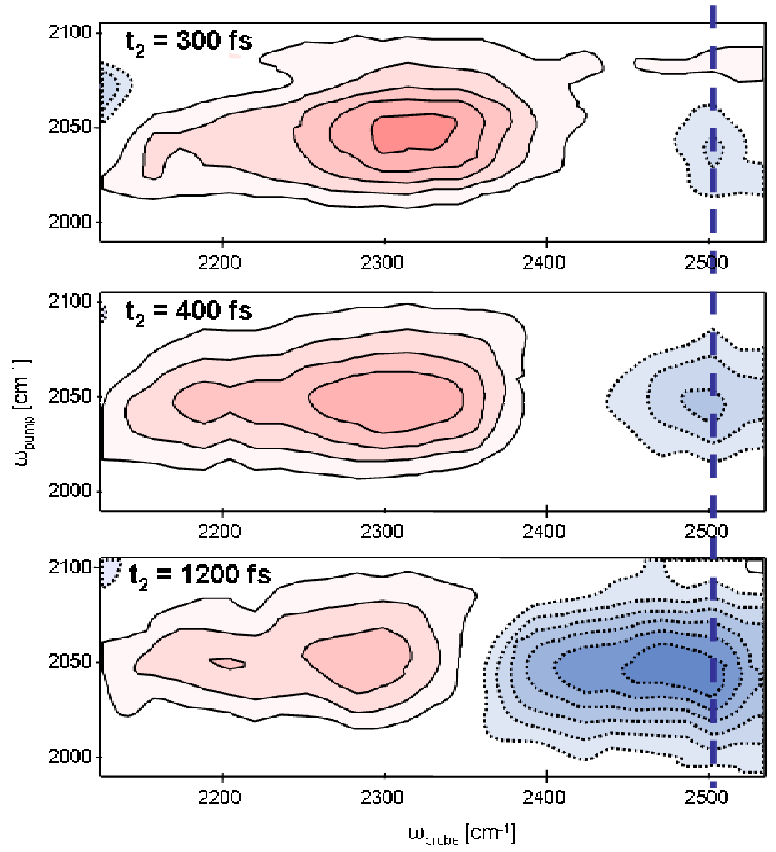

Fig. 1. A. Linear spectrum of $1 \mathrm{M} \mathrm{NaN}$ in $\mathrm{D}_{2} \mathrm{O}$ (solid line) with maximum absorption in the OD-stretch absorption region marked with a dashed line. $\mathrm{D}_{2} \mathrm{O}$ spectrum (dotted line) show for reference. B. 2-color 2D IR spectra of $1 \mathrm{M} \mathrm{NaNa}$ in $\mathrm{D}_{2} \mathrm{O}$ at population times $\mathrm{t}_{2}=300,400$ and 1200 fs. Dotted lobes mark the bleach/stimulated emission signal and the solid lobe - excited state absorption signal. The dashed line marks the maximum of the linear spectrum as in Figure 1A. C. Maximum of the excited state absorption signal in 2D IR spectra as a function of population time $\mathrm{t}_{2}$. Data fitted to a biexponential function giving two time constants, $\tau_{1}=$ $150 \mathrm{fs}$ for the rise and $\tau_{2}=1.7 \mathrm{ps}$ for the decay.

\section{Results and Discussion}

Upon addition of a solute, aqueous hydrogen bond network is perturbed and the changes are reflected in the hydroxyl stretch region. Figure $1 \mathrm{~A}$ shows the linear spectrum of $1 \mathrm{M} \mathrm{NaN} \mathrm{N}_{3}$ in $\mathrm{D}_{2} \mathrm{O}$, in which the addition of solute narrows and blue-shifts the OD-stretch band of heavy water. Moreover, two shoulders at $\sim 2450$ and $\sim 2650 \mathrm{~cm}^{-1}$ are less pronounced. The narrowing is a result of a more defined configuration of water molecules in the presence of a hydrophilic solute, whereas the slight blue-shift of the maximum absorption is a signature of broken hydrogen bonds. However, there is no splitting of the OD-stretch absorption band and no clear distinction between the bulk and solvation water can be made by linear spectroscopy.

Figure 1B shows the measured 2D IR spectra for 3 population times, $\mathrm{t}_{2}=300,400$ and 1200 fs. In the cross-peak shown, the dotted lines represent the ground state absorption and/or bleach signal and the solid lines show the excited state absorption. Additionally, the dashed line indicates the maximum of linear absorption spectrum.

Initially, a narrow bleach signal (FWHM $<130 \mathrm{~cm}^{-1}$ in comparison to $170 \mathrm{~cm}^{-1}$ in linear spectrum) is present, suggesting a selectivity of the method to only a subset of water molecules. i.e. to solvation shell molecules directly coupled to the azide ion. The bleach contribution then grows in width and gains intensity, to dominate the spectra after ca. $1 \mathrm{ps}$ in form of a heat signal. The excited state absorption, on the other hand, is initially a wide and intense signal, which decays with population time $t_{2}$, see Figure 1B and Figure 1C. Another interesting feature is the large off-diagonal 
anharmonicity of ca. $200 \mathrm{~cm}^{-1}$, which suggests the cross-peak is a result of population transfer between the two vibrations rather than a result of direct coupling. The population transfer in this case is an uphill process with a relatively unfavourable maximum probability determined by the energy gap and the Boltzmann factor of ca. $8 \%$.

The timescales of this population transfer can be estimated by fitting the excited state absorption dynamics to a bi-exponential function, as done in Figure 1C. There, two timescales are observed: a fast, $150 \mathrm{fs}$ timescale for the rise of the OD-stretch vibration population and then a decay with a time constant of ca. 1.7 ps. At first, this result seems counter-intuitive since normal timescales for population transfer are of many to tens of picoseconds, however here the timescale that defines the quasi-equilibrium between the population and depopulation of the $\mathrm{D}_{2} \mathrm{O}$ excited state is the population relaxation of $\mathrm{D}_{2} \mathrm{O}$, which was measured to be about 350-400 fs [5]. Due to low population transfer rate, majority of the sample will relax back to ground state via $\mathrm{T}_{1}$ relaxation and the excess energy will be deposited as heat. Therefore, heating effects dominate the spectra much earlier than expected, and after 1 ps the whole sample has thermalized and the selectivity to solvation shell water is lost.

A rather unusual and rare feature is the large difference in relative width of the bleach/ground state absorption vs. excited state absorption contribution. A similar feature has been observed in isotope-diluted ice Ih [6], and to a lesser extent in isotope-diluted water [7]. In ice, this feature has been attributed to the quantum-mechanical delocalization of the vibrational wavefunction in the $\mathrm{R}_{\mathrm{O}} \ldots \mathrm{O}$ direction. Consequently, the spectral width is the measure of the anharmonicity of the hydrogen bond potential and appears to be both similar in ice Ih and azide-bound $\mathrm{D}_{2} \mathrm{O}$. Therefore, we conclude that the hydrogen bond between azide and water has similar anharmonic properties as the hydrogen bond in ice Ih.

\section{Summary and outlook}

We have presented 2-color 2D IR spectra of an intermolecular cross-peak between azide ion and its solvation shell. The observed 2D IR cross-peak is a result of population transfer from azide's asymmetric stretch vibration onto the OD-stretch vibration of $\mathrm{D}_{2} \mathrm{O}$ and thus gives essentially a pump-probe response of solvation shell waters. The population transfer is unusually fast in water, mainly due to the fast relaxation time of liquid water which, as the fastest process, determines the rise time of the cross peak, which was measured to be $150 \mathrm{fs}$. Additionally, it has been argued that the hydrogen bond between azide and water has similar anharmonic features as in ice Ih.

To sum up, the spectroscopy shows a very detailed picture of water-solute hydrogen bond and emerges as a very promising approach for designing higher pulse order experiments, like 3D IR, that would be also able to resolve the inhomogeneity and dynamics of those hydrogen bonds.

\section{References}

1. M. L. Cowan, B. D. Bruner, N. Huse, J. R. Dwyer, B. Chugh, E. T. J. Nibbering, T. Elsaesser, R. J. D. Miller, Nature (London) 434, 199 (2005)

2. M. Ji, M. Odelius, K. J. Gaffney, Science 328, 1003 (2010)

3. C.-H. Kuo, D. Vorobyev, J. Chen, R. M. Hochstrasser, J. Phys. Chem. B 111, 14028 (2007)

4. J. A. Borek, F. Perakis, F. Kläsi, S. Garrett-Roe, P. Hamm, J. Chem. Phys. 136, 224503 (2012)

5. $350-400 \mathrm{fs}$

6. F. Perakis, S. Widmer, P. Hamm, J. Chem. Phys. 134, 204505 (2011)

7. H. J. Bakker, H. K. Nienhuys, Science 297, 587 (2002) 